\title{
ANALYSIS OF TWO COHESIVE ZONE MODELS FOR CRACK PROPAGATION IN NOTCHED BEAMS USING THE BEM
}

\author{
P.C. GONÇALVES ${ }^{1}$, L. PALERMO JR. ${ }^{2}$ \& S.P.B. PROENÇA ${ }^{3}$ \\ ${ }^{1}$ Natural Resources Institute, Federal University of Itajuba, Brazil. \\ ${ }^{2}$ School Civil Engineering, Architecture and Urban Design, University of Campinas, Brazil. \\ ${ }^{3}$ São Carlos School of Engineering, University of São Paulo, Brazil.
}

\begin{abstract}
Crack propagation in a single-edge notched beam is analyzed with the three-point bending test. Two constitutive laws that describe the material softening in the cohesive zone were tested, and their results were compared. The dual boundary element method (DBEM) is employed with the traction boundary integral equation using the tangential differential operator. A constitutive law was introduced in the system of equations, and the cohesive forces were directly computed at each loading step. The results are compared with the experimental and numerical results available in the literature.

Keywords: cohesive model, crack analysis, dual boundary element model, Plane problems, tangential differential operator
\end{abstract}

\section{INTRODUCTION}

A notched specimen of linear elastic material will concentrate stress in the region in front of the notch tip. The development of the damage zone in front of the notch tip is a consequence of these high stresses. The stress distributions in front of a notch, according to Ref. [1], are shown in Fig. 1a for a linear elastic material (curve a) and for a non-yielding material with a micro-cracked zone in front of the notch tip (curve b). Micro-cracks appear in the damage zone of concrete and other non-yielding materials. The material in the fracture or the microcracked zone is partly destroyed but still able to transfer stress. The crack surfaces just behind the fictitious crack tip are not completely separated, and tractions can be transferred across the relatively long extended crack trace, which is called the cohesive zone and is shown in Fig. 1b. The main assumption is that material softening occurs beyond the peak load in a narrow layer with a negligible volume behind the fictitious crack tip in which cohesive forces can stop crack opening. The crack in the cohesive zone can be represented with a two-parameter model named the fictitious crack model. The constitutive laws for the material in the cohesive zone can be defined in terms of stresses and strains accompanied by a layer thickening law or employing a traction-displacement relationship, as adopted in this study. Barenblatt [2] presented a cohesive model using the fictitious crack theory. Hillerborg et al. [3] proposed a function for the softening model related to mode I crack opening (Fig. 1c), which allowed for a finite element model (FEM) analysis of the problem, such as those presented by Petersson [1], Carpinteri [4] and Rots [5].

Several numerical methods besides the FEM have been used to perform crack analyses. The dual boundary element method (DBEM) is one of the most widely used due to its accuracy in computation of the stress intensity factors and the simplicity of adding more elements for crack propagation [6]. The crack analyses for cohesive materials were studied with the DBEM by Saleh and Aliabadi [7, 8], with the Galerkin multizone BEM by Chen et al. [9] and with the displacement discontinuity BEM by Gospodinov [10]. Karlis et al. employed the two dimensional gradient elasticity for crack analyses with the BEM in Ref. [11] and Leonel 


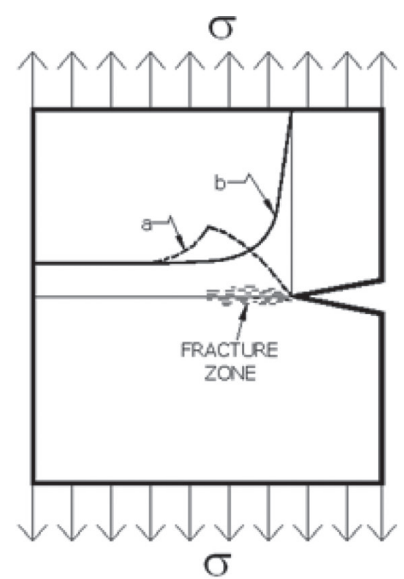

a) Stress Distributions [1]

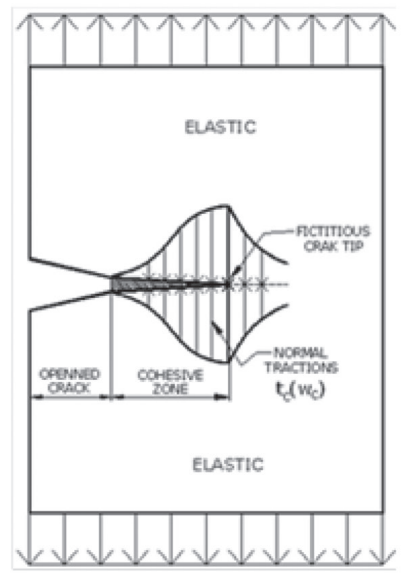

b) Crack in cohesive material [3]

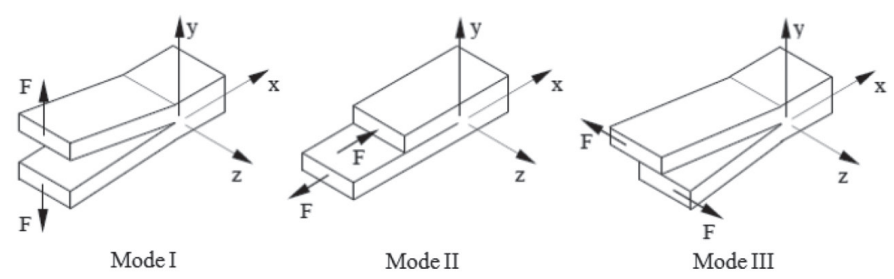

c) Fracture modes

Figure 1: Crack in the cohesive zone.

and Venturini used the tangent operator in the non-linear analyses with a BEM for crack propagation in quasi-brittle materials in Ref. [12]. Tavara et al. performed a BEM analysis of crack propagation along the fiber-matrix interface of laminates using a linear elastic-brittle interface model in Ref. [13]. Trinh et al. employed the fast multipole symmetric Galerkin BEM to solve multizone and multicrack problems in Ref. [14], and Benedetti and Aliabadi employed the BEM in multiscale modeling for polycrystalline materials to analyze material degradation and fracture in Ref. [15].

The DBEM in this study used the tangential differential operator (TDO) in the traction boundary integral equation (BIE). The TDO, in conjunction with integration by parts, is one way to reduce the order of the singularity in kernels of Kelvin-type fundamental solutions and is applied to the hypersingular kernel of the traction BIE here. Kupradze [16] first presented an application with the TDO, and Sladek [17] employed the TDO in a curved crack solution. Bonnet [18] presented regularized formulations of gradients in potential problems by employing the TDO in the BIE, and in stress BIEs for elasticity problems including fracture mechanics formulations. The use of non-conformal interpolations with the TDO requires additional care as explained to the traction BIEs of elasticity for two-dimensional problems [19] and three-dimensional problems [20] and to the stress equations for plate bending including the effect of shear deformation, as in Ref. [21]. 


\section{DUAL BOUNDARY INTEGRAL EQUATIONS FOR COHESIVE MATERIALS}

The coincidence of two crack surfaces requires two different boundary integral equations (BIEs) for the solution and the dual boundary element method (DBEM) is the technique employed. The dual equations of the method are the displacement and the traction boundary integral equations:

$$
\begin{gathered}
\frac{1}{2} u_{i}\left(x^{\prime}\right)+\int T_{i j}\left(x^{\prime}, x\right) u_{j}(x) d(x)=\int U_{i j}\left(x^{\prime}, x\right) t_{j}(x) d(x) \\
\frac{1}{2} t_{k}\left(x^{\prime}\right)=n_{a}\left(x^{\prime}\right) C_{a k i m} \int \sigma_{i b j}\left(x^{\prime}, x\right) D_{m b}\left[u_{j}(x)\right] d(x)-n_{a}\left(x^{\prime}\right) \int \sigma_{j a k}\left(x^{\prime}, x\right) t_{j}(x) d(x)
\end{gathered}
$$

with

$$
\begin{gathered}
C_{a k i m}=\mu\left(\frac{2 v}{1-2 v} \delta_{a k} \delta_{i m}+\delta_{a i} \delta_{k m}+\delta_{a m} \delta_{k i}\right) \\
\mathrm{D}_{b m}[f(x)]=n_{b}(x) f_{, m}(x)-n_{m}(x) f_{, b}(x)
\end{gathered}
$$

where $U_{i j}\left(x^{\prime}, x\right)$ and $T_{i j}\left(x^{\prime}, x\right)$ are the displacement and traction, respectively, in the direction $j$ at boundary point $x$ due to a singular load in the direction $i$ at the collocation point $x^{`}$ based on the Kelvin solution for two-dimensional problems $u_{i}(x)$ and $t_{i}(x)$ are the displacement and traction at boundary point $x$, respectively, $n_{a}\left(x^{\prime}\right)$ is the direction cosine of the outward normal at collocation point $x^{\prime}, C_{a k i m}$ is the Hooke tensor for an isotropic material, $\sigma_{i b j}$ is the stress $\sigma_{b j}$ at boundary point $x$ due to a singular load in the direction $i$ at collocation point $x, D_{b m}($ ) is the tangential operator, $\mu$ is the shear modulus, $v$ is the Poisson ratio, and $\delta_{i j}$ is the Kronecker delta.

The displacement BIE (eqn (1)) is applied to one of the crack surfaces, and the traction BIE (eqn (2)) is applied to the other surface to solve general mixed-mode crack problems with a single domain formulation. Although the integration path is still the same for coincident points on crack surfaces, the respective boundary integral equations are now distinct. The collocation point needed to perform the traction BIE and the strategy used to treat improper integrals are the key features of this formulation. The positions of the collocation points adopted in this study were inside the boundary elements to satisfy the continuity requirements for both BIEs. The left member in eqn (1) has a $1 / r$ singularity, whereas the right member has a logarithmic singularity when the field point approaches the collocation point. Both integrals in the right member of eqn (2) have $1 / r$ singularities because the TDO was used in the first integral.

The cohesive zone is the extension of the crack in which material softening beyond the peak load occurs in the narrow layer behind the fictitious crack tip (Fig. 1b). The cohesive zone is modeled as a crack region connecting coincident surfaces. The points that were originally coincidental on opposite sides of the crack line can separate into distinct points but remain connected by the cohesive zone material. Continued strain increases the separation between these two points and eventually leads to cracking. The simplest approximation for the $\sigma$-w curve in the cohesive zone is a single descending straight line, as shown in Fig ure $2 \mathrm{a}$, where $f_{t}$ is the tensile strength, and $w_{c}$ is the maximum widening of the fracture zone when it is still able to transfer stress. A generalization for the $\sigma-\mathrm{w}$ curve can be made with straightline approximations using an arbitrary number of break points with relations similar to those 

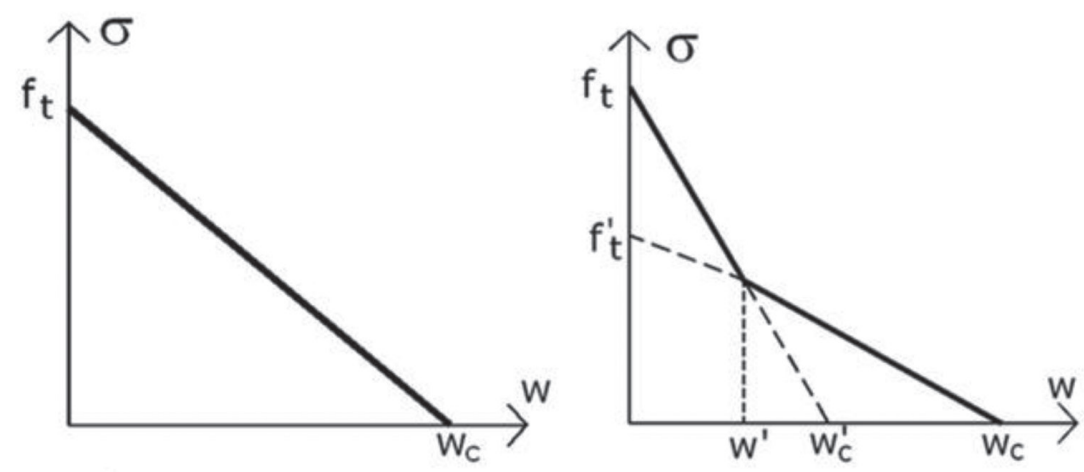

a) $\left\{\begin{array}{c}0 \leq w \leq w_{c} \Rightarrow \sigma(w)=f_{t}\left(1-\frac{w}{w_{c}}\right) \\ w_{c}<w \Rightarrow \sigma(w)=0\end{array}\right.$

b) $\left\{\begin{array}{c}0 \leq w \leq w^{\prime} \Rightarrow \sigma(w)=f_{t}\left(1-\frac{w}{w_{C}^{\prime}}\right) \\ w^{\prime} \leq w \leq w_{C} \Rightarrow \sigma(w)=f^{\prime}{ }_{t}\left(1-\frac{w}{w_{C}}\right) \\ w_{C}<w \Rightarrow \sigma(w)=0\end{array}\right.$

Figure 2: Approximation for a $\sigma-\mathrm{w}$ curve: a) Straight line, b) Two straight lines.

presented in Fig. 2b. The maximum principal stress criterion is used to determine the crack extension increment. When the maximum principal stress at the fictitious crack tip reaches the critical value of $f_{t}$, the tip will extend under further loading using one of the constitutive laws shown in Fig. 2. The tip advances in the direction perpendicular to the maximum principal stress at that point, and the extension is such that the maximum principal stress at the new tip position is equal to the critical $f_{t}$ value during continued loading. The generalized plane stress problem was used in this study to model the beam in a three-point bending test. The maximum principal stress was obtained according to the following expression:

$$
\sigma_{\max }=\frac{1}{2}\left(\sigma_{n}+\sigma_{t}+\sqrt{\left(\sigma_{n}-\sigma_{t}\right)^{2}+4 \tau_{n t}^{2}}\right)
$$

Figure 3 shows a crack containing a cohesive zone. The external boundary line of the problem is $\Gamma_{\mathrm{e}}$, and the tangent direction used to compute the boundary integrals is shown. $\Gamma_{1}$ and $\Gamma_{4}$ are portions of the crack surface without cohesive forces (open crack), while $\Gamma_{2}$ and $\Gamma_{3}$ are

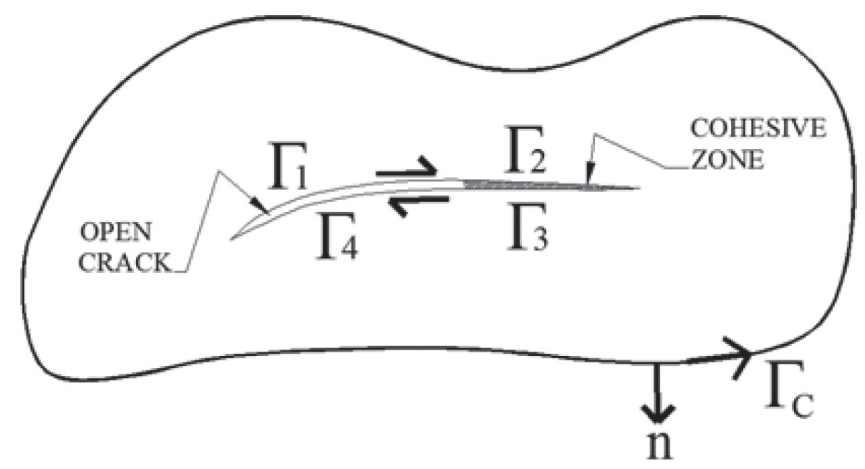

Figure 3: Crack inserted in a finite domain with boundary $\Gamma_{\mathrm{e}}$. 
portions containing cohesive forces. The cohesive zone has tractions on the crack surfaces of opposite sign and equal magnitude. The dual equations for the problem shown in Fig. 3 can be simplified when the directions and magnitudes of the cohesive tractions on crack surfaces $\Gamma_{2}$ and $\Gamma_{3}$ and the directions of integration on these surfaces are taken into account, yielding:

$$
\begin{aligned}
\frac{1}{2} u_{i}\left(x^{\prime}\right)+ & -\int T_{i j}\left(x^{\prime}, x\right) u_{j}(x) d(x)=\int_{e} U_{i j}\left(x^{\prime}, x\right) t_{j}(x) d(x)+2 \int_{c} U_{i j}\left(x^{\prime}, x\right) t_{j}^{c}(x) d(x) \\
\frac{1}{2} t_{k}\left(x^{\prime}\right)= & n_{a}\left(x^{\prime}\right) C_{a k i m} \int \sigma_{i b j}\left(x^{\prime}, x\right) D_{m b}\left[u_{j}(x)\right] d(x)-n_{a}\left(x^{\prime}\right) \int_{e} \sigma_{j a k}\left(x^{\prime}, x\right) t_{j}(x) d(x) \\
& -2 n_{a}\left(x^{\prime}\right) \int \sigma_{j a k}\left(x^{\prime}, x\right) t_{j}^{c}(x) d(x)
\end{aligned}
$$

In eqns (4) and (5), $t^{c}{ }_{j}$ was introduced as the traction in the direction $j$ on one of the crack surfaces within the cohesive zone. The index $c$ was introduced because surface $\Gamma_{2}$ or $\Gamma_{3}$ with tractions $t^{2}$ or $t^{3}$, respectively, can be used. $\Gamma=\Gamma_{1}+\Gamma_{2}+\Gamma_{3}+\Gamma_{4}+\Gamma_{\mathrm{e}}$, and $\Gamma_{2}=\Gamma_{3}=\Gamma_{\mathrm{c}}$.

\section{NUMERICAL IMPLEMENTATION}

The tractions on one of the crack surfaces in the cohesive zone $\left(t_{c}\right)$ are the unknowns in the system of equations obtained from eqns (4) and (5), and the cohesive law is the additional equation used to find the solution. This strategy allows the direct computation of tractions in the cohesive zone at each incremental loading step.

$$
\left[\begin{array}{cccc}
H_{e e} & H_{e o} & H_{e z} & -G_{e z} \\
H_{o e} & H_{o o} & H_{o z} & -G_{o z} \\
H_{z e} & H_{z o} & H_{z z} & -G_{z z} \\
0 & 0 & A & B
\end{array}\right]\left\{\begin{array}{l}
u^{e} \\
u^{o} \\
u^{z} \\
t^{c}
\end{array}\right\}=\left[\begin{array}{cc}
G_{e e} & 0 \\
G_{o e} & 0 \\
G_{z e} & 0 \\
0 & C
\end{array}\right]\left\{\begin{array}{c}
t^{e} \\
w_{f}
\end{array}\right\}
$$

Equation (6) summarizes the system of equations where the dual eqns (4) and (5) are converted to submatrices $H_{i j}$ and $G_{i j}$, and the cohesive law is converted to submatrices $A, B$ and $C$. The indices $e, o$ and $z$ are related to the boundary portions and correspond to the external boundary, the open crack boundary and the cohesive zone, respectively. Submatrices $A$ and $B$ relate the displacements and tractions in the normal and tangent directions, respectively, on the crack surfaces in the cohesive zone.

The softening criterion in the cohesive zone is for tractions and opening in the normal direction, and the tangential tractions are zero. The numerical algorithm is summarized next, considering the boundary portions shown in Fig. 3 and the straight line constitutive law shown in Fig. 2a:

a) The normal tractions $\left(\underline{t}_{c}\right)$ are less than or equal to $f_{t}$, i.e. no opening occurs in the cohesive zone, and opposite points along the crack surfaces have equal displacement. Submatrices $B$ and $C$ are zero, whereas submatrix $A$ contains direction cosines that relate the displacements in the directions $x_{i}$, i.e. the openings in the normal and tangent directions are zero: 


$$
\begin{aligned}
& u_{i}^{2} n_{i}^{2}+u_{i}^{3} n_{i}^{3}=0 \\
& u_{i}^{2} s_{i}^{2}+u_{i}^{3} s_{i}^{3}=0
\end{aligned}
$$

b) With subsequent loads after the normal traction has reached $f_{t}$, opening occurs in the cohesive zone according to the cohesive law as shown in Fig. 2a. The submatrices $B$ and $C$ are then modified to introduce the cohesive law in the line containing the opening in the normal direction. The tractions in the tangent direction become zero, and this condition is introduced in the line describing opening in the tangent direction. Equations (7) and (8) in corresponding lines of matrices $A$ and $B$ are replaced by:

$$
\begin{gathered}
\left(u_{i}^{2} n_{i}^{2}+u_{i}^{3} n_{i}^{3}\right)-\frac{w_{c}}{f_{t}} n_{i}^{c} t_{i}^{c}=-w_{c} \\
s_{i}^{c} t_{i}^{c}=0
\end{gathered}
$$

c) If the opening reaches $w_{c}$ in subsequent loads, the tractions $\left(t_{c}\right)$ must be zero at that point in the cohesive zone. The absence of tractions in the normal and tangent directions causes the tractions in the direction $x_{i}$ to be zero. The tractions $\left(t^{c}{ }_{i}\right)$ at this point on the crack surfaces are eliminated from the system of equations. Equations (9) and (10) in the corresponding lines of matrices $A$ and $B$ are replaced by:

$$
\begin{aligned}
& t_{1}^{c}=0 \\
& t_{2}^{c}=0
\end{aligned}
$$

The break point value ( $\left.w^{\prime}\right)$ is introduced in the numerical algorithm when the constitutive law employs two straight lines, as shown in Fig. 2b. The constitutive law in eqn (9) uses the relation for either the first or second line in Fig. 2b, according to values of the normal opening $(w)$ and the break point ( $\left.w^{\prime}\right)$. The first line is used in the subsequent loading after the normal traction has reached $\mathrm{f}_{\mathrm{t}}$ and opening occurs in the cohesive zone. The second line for the constitutive law replaces the first line when the normal opening value $(w)$ is greater than the break point value $\left(w^{\prime}\right)$. The tractions $\left(\mathrm{t}_{\mathrm{c}}\right)$ must be zero when the normal opening (w) reaches $w_{c}$ in the loading process and eqns (11) and (12) must be used instead of eqns (9) and (10).

The boundary element code was derived from that used in Refs. [22, 23]. Linear mapping functions were used to represent the displacements and tractions in the boundary elements. The same mapping function was used for conformal and non-conformal interpolations, with nodal parameters positioned at the ends of the elements. The collocation points were always positioned on the boundary line at the position $\left(\xi^{\prime}\right)$ in the range $(-1,1)$ : i) $\xi^{\prime}=-0.67$ for continuous elements, and ii) $\xi^{\prime}=-0.67$ and $\xi^{\prime}=+0.67$ for discontinuous elements. Analytical expressions were used to evaluate the singular integrals in the Cauchy principal value, and the Gauss-Legendre scheme was used for regular integrals.

\section{NUMERICAL EXAMPLE}

Experimental and numerical analyses were performed in Ref. [1] for the notched beam under the three-point bending test shown in Fig. 4. The depth of the beam (d) is $0.2 \mathrm{~m}$, the width is $0.05 \mathrm{~m}$ and the length (1) is $2.0 \mathrm{~m}$. The adopted ratio between notched depth and beam depth (a/d) was 0.5. The Young's modulus (E) was $30 \mathrm{GPa}$, the Poisson's ratio (v) was 0.2 and the 


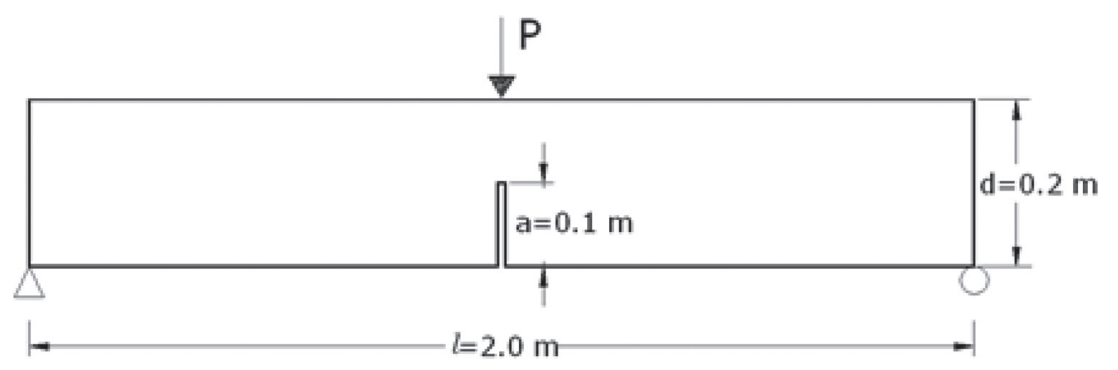

Figure 4: The notched beam subjected to three-point bending.

Table 1: Mechanical properties according to the constitutive laws in Ref. [1].

\begin{tabular}{llllll}
\hline$\sigma-\mathrm{w}$ curve & $G_{f}\left(\mathrm{Nm}^{1}\right)$ & $f_{t}^{\prime}(\mathrm{MPa})$ & $w_{c}\left(10^{-5} \mathrm{~m}\right)$ & $w_{c}^{\prime}\left(10^{-5} \mathrm{~m}\right)$ & $w^{\prime}\left(10^{-5} \mathrm{~m}\right)$ \\
\hline Straight line (SL) & 124 & - & 7.45 & - & - \\
Two straight lines (C) & 124 & 1.427 & 13.40 & 4.468 & 2.979 \\
\hline
\end{tabular}

tensile strength $\mathrm{f}_{\mathrm{t}}$ was $3.33 \mathrm{MPa}$. Table 1 presents the properties used in the analysis of the crack propagation in Ref. [1] for each of the constitutive laws shown in Fig. 2.

Petersson presented results for crack propagation [1], which were obtained experimentally for two values of fracture energy $\left(\mathrm{G}_{\mathrm{f}}\right): 115 \mathrm{Nm}^{1}$ and $137 \mathrm{Nm}^{1}$. Experimental results were compared with those obtained from a numerical analysis [1] with the FEM using $124 \mathrm{Nm}^{1}$ for $\mathrm{G}_{\mathrm{f}}$, which was the median value of the fracture energy values of the experiments. Two constitutive laws were considered in the numerical analyses of Ref. [1]: a) Straight line and b) Two straight lines. Petersson used the letter $\mathrm{C}$ to represent results from the constitutive law using two straight lines because he considered this law better for representing the concrete behavior. The breaking point value ( $w^{\prime}$ ) used in Ref. [1] for the constitutive law with two straight lines was related to the following value of the normal traction:

$$
w^{\prime}=\frac{2}{9} w_{C} \stackrel{\text { yields }}{\rightarrow} \sigma=\frac{1}{3} f_{t}
$$

Saleh and Aliabadi presented results in Ref. [7] for this problem using a DBEM with quadratic boundary elements; the constitutive law in the cohesive zone was the straight line and the mechanical properties were the same employed in Ref. [1]. The results obtained with the DBEM formulation presented here, using TDO and linear boundary elements, are shown in Fig. 5 and compared with those in Ref. [7]. Two types of constitutive laws were used with the present formulation: a single straight line (SL) and two straight lines (C). The same boundary element mesh was used in analyses with both constitutive laws. This analysis employed 196 boundary elements with two boundary nodes placed at each corner and at the fictitious crack tip.

According to Fig. 5, the peak load obtained with a straight line (blue curve) was greater than that obtained with two straight lines (green curve), which were both similar to the result obtained in Ref. [7] with a straight line constitutive law.

A comparison of the experimental and numerical results presented in Ref. [1] is shown in Fig. 6. The results obtained with the present DBEM formulation for both types of constitutive 


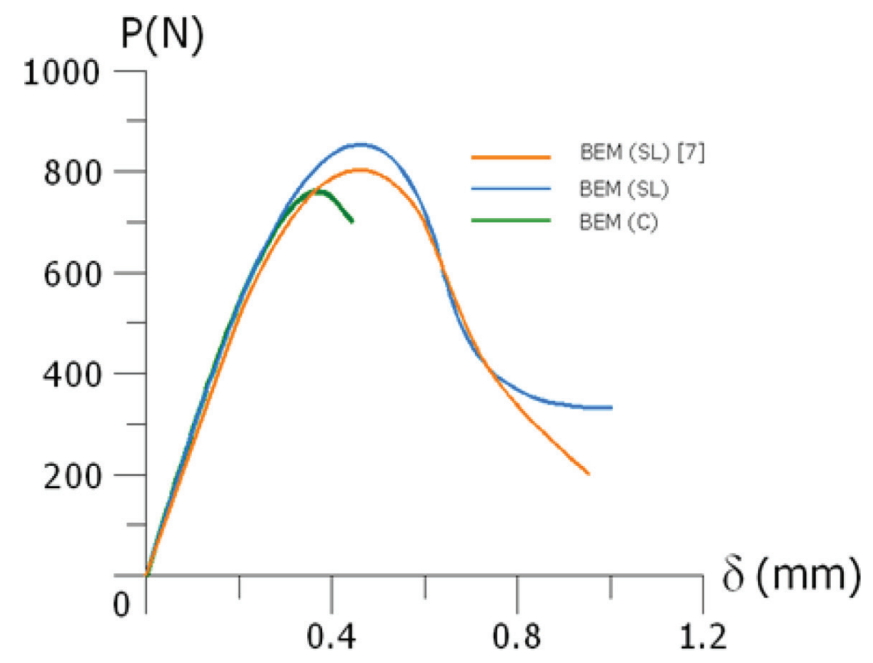

Figure 5: Comparison between the results obtained in this study and those in Ref. [7].

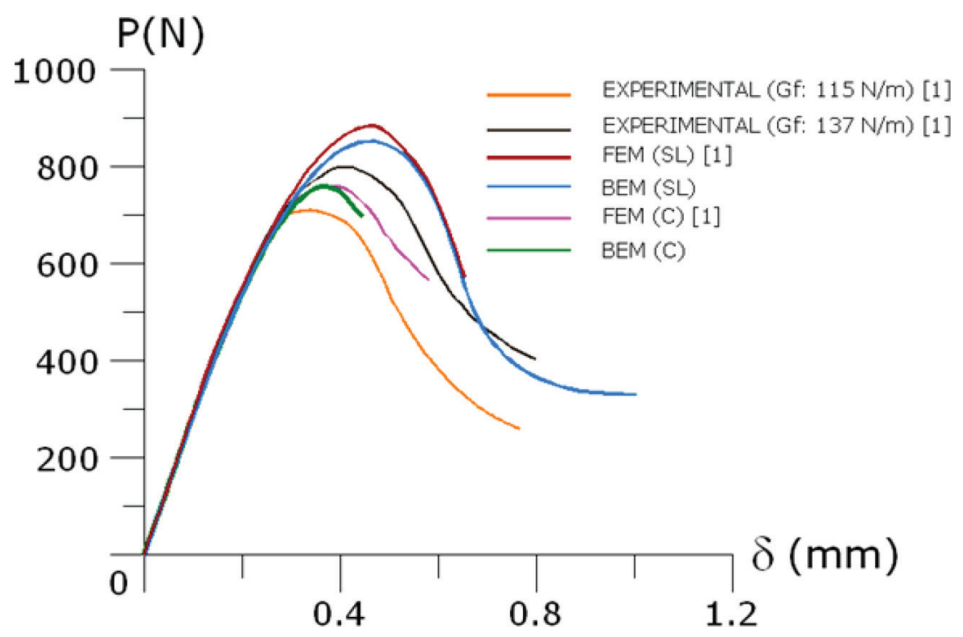

Figure 6: Comparison between the results obtained in this study and in Ref. [1].

laws are very similar to those in Ref. [1], obtained from an FEM analysis. Furthermore, the results obtained with the constitutive law using two straight lines demonstrate that using two straight lines is better to represent concrete behavior, as first noted by Petersson in Ref. [1]. In Fig. 6, the numerical results from the present BEM formulation and FEM [1], using two straight lines for the constitutive law and the median value of fracture energy (pink and green curves), were plotted between the curves obtained experimentally for the upper and the lower value of the fracture energy $\left(\mathrm{G}_{\mathrm{f}}\right)$, which were $137 \mathrm{Nm}^{1}$ (black curve) and $115 \mathrm{Nm}^{1}$ (orange curve). On the other hand, numerical analyses using a straight line for the constitutive law with the median value for the fracture energy $\left(124 \mathrm{Nm}^{1}\right.$ ) presented a peak load (brown and blue curves) greater than that obtained with the experimental analysis (black curve) with the upper value for the fracture energy $\left(137 \mathrm{Nm}^{1}\right)$. 


\section{CONCLUSIONS}

The results obtained in the crack propagation analyses with the present DBEM formulation match those presented in the literature, when either a straight line or two straight lines were used for the constitutive law of the cohesive zone models. Furthermore, the comparison with earlier results presented by Petersson [1] confirmed his conclusions, which were based on experimental tests and numerical analyses using an FEM. The model formulation used in this study, which was presented in Ref. [22], was more stable and computationally faster than the method presented in Ref. [24], as noted in Ref. [23].

\section{REFERENCES}

[1] Peterson, P.E., Crack growth and development of Fracture Zone in Plain Concrete and similar materials. Report No TVBM-1006, Division of Building Materials, Lund Institute of Technology, Lund Sweden, 1981.

[2] Barenblatt, G.I., The mathematical theory of equilibrium cracks in brittle fracture. Advances in Applied Mechanics, 7, pp. 55-129, 1962.

https://doi.org/10.1016/s0065-2156(08)70121-2

[3] Hillerborg, A., Modeer, M. \& Petersson, P.E., Analysis of crack formation and crack growth in concrete by means of fracture mechanics and finite elements. Cements Concrete Research, 6, pp. 773-782, 1976. https://doi.org/10.1016/0008-8846(76)90007-7

[4] Carpinteri, A., Post-peak and post-bifurcation analysis of cohesive crack propagation. Engineering Fracture Mechanics, 32, pp. 265-278, 1989. https://doi.org/10.1016/0013-7944(89)90299-3

[5] Rots J.G., Computational modeling of concrete fracture. Ph.D. Thesis, Delft University of Technology, 1988.

[6] Brebbia, C.A., The birth of the boundary element method from conception to application. Engineering Analysis with Boundary Elements, 77, pp. iii-x, 2017. https://doi.org/10.1016/j.enganabound.2016.12.001

[7] Saleh, A.L. \& Aliabadi, M.H., Crack growth analysis in concrete using boundary element method. Engineering Fracture Mechanics, 51(4), pp. 533-545, 1995. https://doi.org/10.1016/0013-7944(94)00301-w

[8] Saleh, A.L. \& Aliabadi, M.H., Fracture Mechanics Analysis of crack on plain concrete using the boundary element methods. Engineering Fracture Mechanics, 69, pp. 267-280, 2002. https://doi.org/10.1016/s0013-7944(01)00089-3

[9] Chen T, Wang B, Cen Z, Wu Z., A symmetric Galerkin multi-zone boundary elemento method for cohesive crack growth. Engineering Fracture Mechanics, 63, pp. 591-609, 1999.

[10] Gospodinov, G., Boundary element modelling of cohesive cracks using displacement discontinuity method. Advances in Boundary Element Techniques, pp. 19-26, 2003.

[11] Karlis, G.F., Tsinopoulos, S.V., Polyzos, D. \& Beskos, D.E., Boundary Element analysis of mode I and mixed mode (I and II) crack problems of 2-D gradient elasticity. Computer Methods in Applied Mechanics and Engineering, 196, pp. 5092-5103, 2007. https://doi.org/10.1016/j.cma.2007.07.006

[12] Leonel, E.D. \& Venturini, W.S., Non-linear boundary element formulation with tangent operator to analyze crack propagation in quasi-brittle materials. Engineering Analysis with Boundary Elements, 34, pp. 122-129, 2010.

https://doi.org/10.1016/j.enganabound.2009.08.005 
[13] Távara, L., Mantic, V., Graciani, E. \& París, F., BEM analysis of crack onset and propagation along fiber-matrix interface under transverse tension using a linear elastic-brittle interface model. Engineering Analysis with Boundary Elements, 35, pp. 207-222, 2011. https://doi.org/10.1016/j.enganabound.2010.08.006

[14] Trinh, Q.T., Mouhoubi, S., Chazallon, C. \& Bonnet, M., Solving multizone and multicrack elastostatic problems: a fast multipole symmetric Galerkin boundary element method approach. Engineering Analysis with Boundary Elements, 50, pp. 486-495, 2015. https://doi.org/10.1016/j.enganabound.2014.10.004

[15] Benedetti, I. \& Aliabad, M.H., Multiscale modeling of polycrystalline materials: a boundary element approach to material degradation and fracture. Computer Methods in Applied Mechanics and Engineering, 289, pp. 429-453, 2015. https://doi.org/10.1016/j.cma.2015.02.018

[16] Kupradze, V.D., Three-dimensional problems of the mathematical theory of elasticity and thermoelasticity. North Holand, 1979.

[17] Sladek, J. \& Sladek, V., Three-dimensional curved crack in an elastic body. International Journal of Solids and Structures, 19, pp. 425-436, 1983. https://doi.org/10.1016/0020-7683(83)90053-7

[18] Bonnet, M., Boundary Integral Equation Methods for Solids and Fluids, John Wiley \& Sons Ltd, 1999.

[19] Palermo, Jr., L., Almeida, L.P.C.P.F. \& Gonçalves, P.C., The use of the tangential differential operator in the dual boundary element equation. Structural Durability \& Health Monitoring, 2(2), pp. 123-130, 2006.

[20] Palermo, Jr., L. \& Almeida, L.P.C.P.F., On the use of the tangential differential operator in the traction boundary integral equation of the dual boundary element method for three dimensional problems. ICCES, 7(2), pp. 83-87, 2008.

[21] Palermo, Jr., L., The tangential differential operator applied to a stress boundary integral equation for plate bending including the shear deformation effect. Engineering Analysis with Boundary Elements, 36, pp. 1213-1225, 2012.

https://doi.org/10.1016/j.enganabound.2012.02.010

[22] Gonçalves, P.C., Aplicação do método dos elementos de contorno no estudo da propagação de fissura discreta para modelos coesivos ( $\mathrm{PhD}$ thesis, written in Portuguese), 136p., Faculdade de Engenharia Civil, Arquitetura e Urbanismo, Universidade Estadual de Campinas, 2015.

[23] Gonçalves, P.C., Palermo, L. \& Proenca, S.P.B., A boundary element formulation for crack analyses incorporating a cohesive zone model. International Journal of Computational Methods and Experimental Measurements, 5(3), pp. 231-240, 2017. https://doi.org/10.2495/cmem-v5-n3-231-240

[24] Gonçalves, P.C., Figueiredo, L.G., Palermo Jr., L. \& Proença, S.P.B., The Dual-Boundary-Element Formulation Using the Tangential Differential Operator and Incorporating a Cohesive Zone Model for Elastostatic Cracks. 12th International Conference Boundary Element and Meshless Techniques, Brasília, 2011. 